\title{
Procesos de capacitación ayudados por TIC en el ámbito laboral
}

\author{
Yohanna Abarca Amador \\ Escuela de Ciencias del Lenguaje \\ Instituto Tecnológico de Costa Rica
}

\begin{abstract}
Resumen
Este estudio discute cómo los procesos de capacitación han evolucionado influenciados por la historia, la psicología, la educación y, más recientemente, la tecnología. Estos procedimientos son necesarios para maximizar la eficiencia del recurso humano, el más valioso de un país. En la actualidad, muchas compañías e instituciones del sector público y del privado apuestan por la enseñanza por medio de las TIC (Tecnologías de la Información y la Comunicación). El artículo describe algunas perspectivas que han moldeado el aprendizaje apoyado por las TIC a través del tiempo. Además, se incluyen tres experiencias de formación de este estilo en diferentes instituciones con sus ejemplos correspondientes.
\end{abstract}

Palabras claves: procesos de capacitación, sector público, sector privado, tecnologías de la información y comunicación (TIC), experiencias de capacitación con TIC

\begin{abstract}
This study discusses the evolution of the training processes under the influence of history, psychology, education, and more recently, technology. These processes are necessary to maximize the efficiency of the human resource, a country's most valuable resource. Therefore, nowadays many companies and institutions from the public and private sector are taking bets on the training processes by means of the IT. The study also discusses some perspectives which have given shape to the training processes through time. Three experiences of training processes aided by IT are also described with their corresponding examples.
\end{abstract}


Key words: training processes, public sector, private sector, information technologies (IT), training experiences with IT, related examples

\section{Introducción}

$\mathrm{E}$ 1 estudio y la aplicación de los procesos de capacitación en el ámbito laboral han ido variando para responder a las diversas perspectivas y teorías que los han permeado. Así pues, estos han cambiado paulatinamente a causa de las guerras, las distintas corrientes que han influenciado la psicología y la educación, las transformaciones que se han generado en la economía mundial a través de la historia, y los avances tecnológicos. Estos cambios han sido necesarios con el fin de incrementar la eficiencia de los procesos y la calidad de los resultados en las industrias o empresas grandes o pequeñas, estatales o del sector privado. Sin embargo, para que esto sea posible, es necesario apostar al potencial humano que es el más valioso recurso de un país. Muchos proyectos y programas se han desarrollado en diversos países con el fin de mejorar y fomentar la capacitación de la fuerza laboral. No obstante, estos esfuerzos deben continuar e incrementarse si se desea tener un resultado más positivo que lleve al desarrollo de los países, especialmente en los que las Tecnologías de la Información y la Comunicación (TIC) han tenido un avance más lento.

En la actualidad, el uso de las TIC ofrece valiosas oportunidades de capacitación a los trabajadores de todos los sectores productivos de un país. Según Alemán y Gómez (2011), ante los nuevos retos que plantea la globalización, las empresas e instituciones en general se ven ante la necesidad de enfrentar la caducidad de sus servicios y productos. Por ende, para que estos permanezcan competitivos, tanto empresas como instituciones deben realizar transformaciones continuas en el contenido de los perfiles de trabajo, las cambiantes demandas del mercado y las competencias necesarias por parte de los trabajadores en todos los niveles jerárquicos. En este contexto, las TIC son una herramienta indispensable para lograr vigencia en el mercado y poder ofrecer servicios y productos de calidad, ya que ayudan a implementar diversos procesos de capacitación que de otro modo no podrían llevarse a cabo en empresas e instituciones. Nuestro país no se escapa de esta realidad. Con la implementación de mejores procesos de capacitación en el ámbito laboral se podrían mejorar servicios al tiempo que se brindarían opciones de actualización profesional a los trabajadores.

A continuación, se presenta una breve reseña de las diversas perspectivas y teorías que han moldeado los procesos de capacitación en el campo laboral a través del tiempo. Asimismo, se indican cuáles son las cuatro etapas que se deben seguir en todo proceso de capacitación con TIC. Por último, se incluyen tres ejemplos de experiencias de capacitación llevados a cabo de forma exitosa en distintos países. 


\section{Perspectivas y teorías sobre los procesos de capacitación}

Para Alemán y Gómez (2011), el estudio de la capacitación laboral ha evolucionado a lo largo del tiempo mediante tres perspectivas: la histórica, la sociológica y la económica. En cuanto a la histórica, los cambios en la economía y la sociedad impulsaron un modelo de desarrollo enfocado en la producción. A partir de ahí, la educación continua en los adultos cobró importancia. Ya no era suficiente con tener un oficio, sino que se debían actualizar constantemente los conocimientos y competencias. Por ejemplo, los principios "tayloristas", desarrollados por el estadounidense Frederick Winston Taylor a principios del siglo XX en su libro The Principles of Scientific Management (1911), fomentaron el uso del currículo como un medio observable o de medición de las capacidades adquiridas por medio de la instrucción. Bajo estos principios, el currículo medía los avances del trabajador en el conocimiento requerido para desarrollarse apropiadamente en su puesto laboral (Taylor, citado por Coriat, 1991).

Según la doctrina "taylorista", los patronos debían tomar conciencia de que un empleado bien entrenado y con mejores conocimientos representaba una ventaja para la empresa. Taylor sustentaba la perspectiva científica de que la administración daría un nuevo giro a la manera de cómo se hacía el trabajo en aquella época. De esta forma, las personas que administraban la producción debían adquirir nuevas responsabilidades. En consecuencia, se empezaron a desarrollar talleres artesanales para promover la relación entre trabajo y formación. Más adelante, se evolucionó de academias a escuelas superiores y escuelas-fábricas hasta que se llegó a establecer la idea de "cursos" para capacitar a los nuevos empleados. Tal evolución favoreció la eficiencia y el crecimiento de la empresa. En suma, el deseo de Taylor en aplicar su "gerencia científica" iba en la noble dirección de conseguir la máxima prosperidad del empresario y el trabajador.

Desde la perspectiva sociológica, se ha estudiado la relación existente entre las estructuras que forman una empresa y la capacitación, especialmente a partir del surgimiento de las micro, pequeñas y medianas empresas. Muchos factores pueden determinar la capacitación requerida para una empresa: desde el número de empleados hasta la regulación de esta por parte del Estado. Por tanto, se busca que exista una política pública donde la formación esté inmersa dentro de un proceso social. En este sentido, los procesos instructivos no se ven solo como inversiones particulares que favorecerán a un individuo o una compañía en particular, sino como un elemento que beneficiará a la sociedad en general.

La perspectiva económica promueve, por su parte, la capacitación en grandes y pequeñas empresas. Durante el entrenamiento, se da énfasis al comportamiento del mercado con el fin de conocer qué productos o servicios son necesarios. La formación se ve como una inversión inmediata con el objetivo de obtener beneficios monetarios futuros, no solo en el nivel personal en el caso del empleado, sino también de las mismas empresas; en última instancia, la capacitación se proyecta además a la sociedad en general.

Finalmente, no se debe dejar de lado un factor histórico importante: la experiencia generada después de las 
dos guerras mundiales. En varios países europeos, como resultado de ambos eventos, se adoptó la idea de que la capacitación se debía realizar dentro de la empresa y por supervisores entrenados en las labores cotidianas de las fábricas nacientes. En otras palabras, los capacitadores imitaron en lo laboral lo que se había puesto en práctica en el campo militar (Alemán y Gómez, 2011).

Los enfoques teóricos que se han aplicado también han ido cambiando a través del tiempo. Según Alemán y Gómez, han surgido distintas teorías provenientes de diversos campos con aportes valiosos a los procesos de capacitación. Por tanto, desde el punto de vista de la administración de los procesos de capacitación, se han desarrollado teorías que explican la función del recurso humano de acuerdo con la estructura, la tarea y las relaciones humanas: (1) la Teoría Clásica explica cómo ser eficiente mediante la mecanización de funciones; (2) el Humanismo hace énfasis en la necesidad de la motivación de los subordinados para que sean más eficientes; (3) la Teoría Estructuralista intenta que los trabajadores interioricen sus obligaciones para que las cumplan de forma voluntaria y con una actitud positiva; (4) la Teoría Neoclásica enfatiza la importancia del planeamiento para la obtención de mejores resultados tanto en el proceso de capacitación como posteriormente en la práctica laboral; y (5) el Conductismo intenta generar aprendizajes y comportamientos en el individuo para organizar esfuerzos que se puedan integrar en los grupos. Estas teorías han sentado las bases para los procesos de capacitación en la actualidad.

En este punto, conviene mencionar la importancia de la Teoría General
Sistémica del austriaco Ludwig Bertalanffy. Esta propone que la práctica cotidiana de sus labores debe ser estimulante para el trabajador. Según su teoría, las propiedades de los sistemas no pueden describirse significativamente en términos de sus elementos separados. La comprensión de los sistemas ocurre únicamente cuando se estudian globalmente, involucrando todas las interdependencias de sus partes. Bertalanffy enmarca la capacitación de forma integral, junto con todos los otros componentes de una organización, a saber: la jerarquía, el recurso humano, la especialidad y el área de trabajo (Bertalanffy, 1969).

Asimismo, dentro de la pedagogía y la psicología, destaca el Pragmatismo de John Dewey que se centra en los procesos de socialización y reproducción de la cultura como los fundamentos de todo proceso educativo. En la perspectiva de Dewey, la capacitación se debe ver enmarcada dentro de los cánones culturales de la sociedad en que se lleve a cabo (Westbrook, 1993). Se aprende entonces haciendo y resolviendo problemas concretos y personales, y no escuchando. Dewey sostenía que, para llegar a la finalidad formativa del conocimiento, habría de pasarse por tres etapas primordiales: (1) los hechos y acontecimientos científicos, (2) las ideas y razonamientos, y (3) la aplicación de los resultados a nuevos hechos o acontecimientos. Para Dewey, la aplicación de los conocimientos teóricos en la práctica en el entorno laboral es el punto de partida para un efectivo desarrollo de los procesos de formación.

Finalmente, es importante referirse al "tecnicismo" de la teoría de Bobbit (1918), la cual propone que la educación es una herramienta que se 
debe adaptar a las necesidades de los individuos y de la sociedad. Por tanto, se deben aprender solamente aquellas habilidades que sean necesarias para llevar a cabo de forma eficiente las labores que den respuesta a las demandas del mercado laboral. En la sociedad actual, el pragmatismo de esta teoría sigue teniendo auge entre quienes ven en la educación una herramienta para acceder a la fuerza laboral. Además, algunas instituciones en el ámbito público se encargan de actualizar e informar a la sociedad sobre las demandas del mercado con respecto a los conocimientos y habilidades que los trabajadores deberán poseer para poder insertarse exitosamente en el ámbito laboral.

Las teorías arriba mencionadas han servido de marco conceptual a través del tiempo para implementar diversos procesos de capacitación. Estos procedimientos están en continuo cambio, según Alemán y Gómez, ya que promueven el desarrollo personal y organizacional de acuerdo con las necesidades presentes y futuras de la empresa, el mercado y la sociedad. Además, la capacitación está integrada por ciclos en los cuales, como señala Chiavenato (1985), se conjugan procesos administrativos y pedagógicos. Por esta razón, las experiencias de cada país con respecto a los procesos instructivos son diversas. Es decir, estas incluyen desde las necesidades de cada empresa en particular hasta las fuentes de financiamiento que apoyan estos procesos.

\section{Etapas de los procesos de capacitación ayudados por TIC}

Los procesos de capacitación ayudados por TIC requieren del cumplimiento de cuatro etapas que se describen a continuación.

\section{Primera etapa: diagnóstico de necesidades de capacitación (DNC)}

La primera etapa está comprendida por el diagnóstico de necesidades. En ella, se toman en cuenta las demandas de la empresa y el personal con respecto a capacitaciones que les lleven a realizar funciones más complejas en el presente o el futuro. Al respecto, Méndez (2004) afirma que la formación es un medio para lograr un objetivo determinado. Por tanto, el primer paso para alcanzar este objetivo es identificar las necesidades de capacitación en cada empresa. Esto se logra, en primera instancia, al realizar una evaluación profunda y concienzuda del lugar de trabajo por medio de un diagnóstico de necesidades de capacitación (DNC). Aguilar (2010) indica que el objetivo principal del DNC es identificar las diferencias entre las capacidades y competencias que poseen los trabajadores de la empresa para enfrentar las demandas del mercado. Así, el DNC permite reconocer los departamentos o el personal que necesitan ser capacitados y qué tipo de capacitación requieren. En cuanto al lugar donde las capacitaciones deben llevarse a cabo, Alemán y Gómez proponen que esta tarea se debe realizar en cada unidad y puesto de trabajo. 


\section{Segunda etapa: plan maestro de capacitación}

La segunda etapa consiste en el diseño y elaboración del plan maestro de capacitación propiamente dicho. En esta fase, se establecen claramente los objetivos generales y específicos por cumplir mediante el proceso de capacitación. A partir de ahí, se realiza la planeación operativa, es decir, se define quiénes serán capacitados, por quién y por cuánto tiempo.

\section{Tercera etapa: programa de capacitación}

En la tercera fase, se ejecuta el programa de capacitación, el cual determina las estrategias necesarias para llevar a cabo las actividades. Además, se establece cuál será la fuente de financiamiento.

\section{Cuarta etapa: evaluación del proceso de capacitación}

En la cuarta fase, se evalúan los resultados de la capacitación. Esta etapa es crucial pues constituye el periodo en el que (1) se le da seguimiento al proceso, (2) se determina si los resultados obtenidos son los esperados y (3) se señalan los aspectos del programa que se deben mejorar o cambiar en el futuro.

\section{Ejemplos de capacitaciones ayudadas por las TIC}

A continuación, se describen tres ejemplos de capacitación apoyada por TIC y cómo se dio cada uno según la etapa.

\section{Ejemplo 1}

Alonso y Zamora (2007) describen el ejemplo 1 denominado: "Utilización de las Tecnologías de la Información y las Comunicaciones (TIC) en la Formación y Desarrollo del Capital Humano: la Experiencia de la Empresa de Telecomunicaciones de Cuba, S.A. (Etecsa)".

\section{Primera etapa: diagnóstico de necesidades de capacitación (DNC)}

Todos los niveles o departamentos de trabajo requerían de capacitación para poder desarrollar su labor de forma eficiente. Dependiendo del puesto de trabajo, cada individuo debía llevar distintos cursos de capacitación. Sin embargo, al tener Etecsa oficinas en distintas partes del país, no todos los trabajadores podían recibir la capacitación que se realizaba de forma presencial; esto generaba asimismo grandes costos de transporte, hospedaje y alimentación. Además, debido a que algunos cursos tomaban hasta dos meses, no todos los trabajadores podían desplazarse al sitio donde estos se impartían. Estas condiciones crearon la necesidad de que los trabajadores pudieran acceder a cursos de capacitación en línea o ayudados por TIC. Se ofrecieron cursos en línea en las temáticas de conmutación y tráfico telefónico, pizarras privadas, transmisión, planta exterior y redes de acceso, telefonía pública, telefonía móvil, redes de datos, informática, y recursos humanos. En total, se ofrecieron aproximadamente cien cursos en un solo año a más de mil trescientos alumnos de la compañía. 


\section{Segunda etapa: plan maestro de capacitación}

Debido a las necesidades de capacitación del personal y a los problemas que generaban los cursos presenciales, se estableció como meta la creación de la Unidad de Teleformación para ofrecer de forma continua cursos en línea. A partir de este objetivo, se llevaron a cabo las siguientes acciones de capacitación:

1. Se diagnosticó la infraestructura tecnológica disponible en la empresa y se decidió invertir en la adquisición una plataforma virtual.

2. Se definió el modelo educativo para el proceso de enseñanza de la empresa, poniendo énfasis en el autoaprendizaje y la colaboración. Además, se promovieron cambios en la cultura empresarial respecto al uso de las TIC en actividades de capacitación.

\section{Tercera etapa: programas de capacitación}

En esta fase, se obtuvo el apoyo de la dirección de la empresa para poder financiar en forma continua los cursos en línea. Se plantearon, igualmente, tres objetivos específicos para el desarrollo de estos cursos:

1. Atender las necesidades de capacitación de quienes se les imposibilita la asistencia al Centro de Formación y abarcar, de esta manera, una población más amplia de trabajadores.

2. Disminuir costos de transporte y estadía.

3. Involucrar a profesores y conferencistas en los cursos virtuales que de otra manera no estarían disponibles.
A partir de estos propósitos, se cumplieron las siguientes actividades de capacitación.

1. Se realizó una capacitación inicial en el uso de la plataforma virtual para el personal de la Unidad de Teleformación y para los docentes del Centro de Formación. También, se entrenó a los capacitadores en temas relacionados con el uso de las TIC en el proceso de enseñanzaaprendizaje, así como en el diseño de cursos para entornos virtuales de formación.

2. Se puso a la disposición de los trabajadores seminarios online y cursos desarrollados por el Claustro Docente y Entrenadores Adjuntos para la especialización o superación del personal.

3. Además, se ofrecieron cursos desarrollados por proveedores nacionales y extranjeros pertinentes a las temáticas de la empresa.

\section{Cuarta etapa: evaluación del proceso de capacitación}

En esta, se le dio seguimiento al desarrollo de los cursos, la cobertura que estos tuvieron en los trabajadores y el impacto generado en la empresa. A partir de esta valoración, se generaron cambios.

\section{Ejemplo 2}

La segunda experiencia es un proyecto en el sector educativo que se presentó para el premio ABA (Asociación de Bancos Argentinos). Esta Asociación busca premiar proyectos que incentiven la inserción laboral por medio de tecnologías. La experiencia fue desarrollada por Zega y Moreno, quienes 
la describieron de la siguiente forma: "Experiencia de capacitación docente en recursos de comunicación mediada por TIC y capacitación para integrar TIC al currículo de la cátedra universitaria: CHeTIC (Curso de Posgrado de Herramientas Tic en el marco de la web) y PresTIC (Curso de Posgrado para emprender cambios en la integración de las TIC): dos cursos en línea desarrollados para la Facultad de Ciencias Económicas y Empresariales de la Universidad Morón de Buenos Aires".

\section{Primera etapa: diagnóstico de necesidades de capacitación (DNC)}

Esta experiencia se llevó a cabo en el sector educativo en el nivel de formación de profesores de la Facultad de Ciencias Económicas y Empresariales de dicha universidad. De acuerdo con Zega y Moreno (2011), muchos docentes de la Universidad Morón y beneficiarios de la experiencia de capacitación gestionaron las TIC para su uso personal y capacitación profesional.

El estudio reflejó que existía un divorcio entre lo que se consideraba el uso personal de las TIC y su integración a la docencia. Además, la mayoría de profesores era abogados, contadores, administradores de empresas y psicólogos, entre otros. Estos educadores no estaban al tanto de las nuevas metodologías didácticas, por lo que terminaban dando prioridad a la enseñanza o transmisión de conocimientos antes que al aprendizaje. Con la apertura de carreras a distancia, se hizo patente la necesidad de que estos profesores recibieran la debida capacitación pedagógica para así desenvolverse con éxito en ambientes en línea.

\section{Segunda etapa: plan maestro de capacitación}

Dos cursos fueron propuestos para cubrir las necesidades de capacitación docente en la universidad:

El CHeTIC es la sigla del Curso sobre Herramientas de las Tecnologías de la Información y la Comunicación. Este constituye el primer nivel de esta propuesta educativa que responde al requisito de la Facultad en su plan de formación docente e innovación educativa. El segundo nivel PresTIC se relaciona con el uso de las TIC para apoyar los programas de formación en el desarrollo de competencias docentes que permitan la inclusión de TIC en las cátedras presenciales para llevar a cabo diversos procesos formativos en currículos de la educación superior.

La Facultad tenía como objetivo general que sus docentes tuvieran una capacitación más profunda con el fin de poder utilizar TIC como recursos en sus clases y poder implementar cursos a distancia dentro de las carreras ofrecidas. Para alcanzar este propósito, se realizaron las siguientes acciones de capacitación:

1. Se desarrollaron los dos cursos arriba mencionados (CHeTIC y PresTIC), los cuales se convirtieron en requisito para poder impartir cursos a distancia.

2. Se organizaron grupos de entre 28 y 36 docentes para llevar el curso.

3. Se otorgaron becas a todos los docentes interesados en llevar los dos cursos. 


\section{Tercera etapa: programas de capacitación}

Los programas de capacitación se desarrollaron de acuerdo con los siguientes objetivos:

1. Apropiar, actualizar y profundizar el conocimiento y buen manejo de las Tecnologías de la Información y Comunicación.

2. Lograr el aprendizaje y la apropiación de metodologías y recursos didácticos, comunicacionales y tecnológicos para integrar exitosamente las Tecnologías de la Información y Comunicación al currículo.

3. Emprender cambios en la integración de TIC a las cátedras presenciales, así como diseñar módulos de aprendizaje virtual.

Los cursos CHeTIC y PresTIC se basaron en las siguientes actividades:

1. Elaboración colaborativa de conocimientos entre los participantes de ambos cursos.

2. Ofrecimiento de recursos visuales y textuales, como participación en foros de discusión junto a ayudas, tutoriales y herramientas tecnológicas.

\section{Cuarta etapa: evaluación del proceso de capacitación}

Para evaluar el proceso de capacitación se utilizaron las TIC como un medio para incentivar la reflexión sobre temas académicos y teóricos. Además se realizó una evaluación formativa y una sumativa sobre diversos aspectos del desempeño de cada docente al final de cada curso.

\section{Ejemplo 3}

De acuerdo con Castañón (2010), el ejemplo 3 que se describe a continuación corresponde a la propuesta de trabajo implementada en la Universidad Privada Og Mandino en Quito, Ecuador, por medio de la empresa de capacitación Expertos en Entornos Virtuales Tecnología con Metodología. El nombre de esta capacitación fue Implementación del Programa de Capacitación Docente en el Correcto Uso de las TIC en la Universidad Og Mandino en el año 2010 a través de la Aplicación de la Metodología Pacie (Presencia, Alcance, Capacitación, Interacción y ELearning). Esta metodología se utiliza para el uso y aplicación de herramientas virtuales (Oñate, 2009).

\section{Primera etapa: diagnóstico de necesidades de capacitación (DNC)}

Esta propuesta se centró en el sector docente de la Universidad Og Mandino. La Universidad cuenta con una plataforma como soporte tecnológico adecuado y cada docente tiene acceso a un aula virtual. Sin embargo, las herramientas están subutilizadas, según el análisis FODA, debido al desconocimiento del personal docente acerca del manejo de la plataforma y de las posibilidades que ofrece.

A partir de esta problemática surgió la necesidad de crear un programa de capacitación docente institucional en el uso correcto de las TIC. El propósito del programa era desarrollar las destrezas cognitivas, afectivas y psicomotrices necesarias para la gestión y manejo apropiado de recursos en entornos o aulas virtuales a partir del desarrollo de diversas actividades dentro del propio campus virtual. 


\section{Segunda etapa: plan maestro de capacitación}

La finalidad general del programa era capacitar al personal docente de la Universidad en el uso correcto de las TIC con la Metodología Pacie. Para lograrlo, se implementaron tres cursos de capacitación al personal docente: Entornos Virtuales de Aprendizaje (EVA), Recursos Educativos Virtuales y Metodología Pacie. Estos cursos tenían la finalidad de fomentar el uso adecuado de las TIC dentro de los entornos virtuales.

\section{Tercera etapa: programas de capacitación}

La capacitación se basó en los siguientes objetivos específicos, los cuales sirvieron como base para la capacitación de los docentes:

1. Generar procesos de autoaprendizaje de Entornos Virtuales de Aprendizaje (EVA).

2. Emplear con destreza Recursos de Entornos Virtuales.

3. Aplicar adecuadamente la Metodología Pacie en las aulas virtuales de aprendizaje.

\section{Cuarta etapa: evaluación del proceso de capacitación}

En esta última fase, se evaluaron la aplicación del programa y los resultados obtenidos de su aplicación en la Universidad Og Mandino con el fin de contabilizar las mejoras y los aspectos por corregir. El análisis de los resultados obtenidos se presentó mediante una aplicación del programa en forma virtual.

\section{Conclusión}

Los procesos de capacitación han evolucionado a través del tiempo debido a cambios en la economía mundial y la tecnología. Estas transformaciones han dictado pautas cada vez más diversas sobre las necesidades de capacitación laboral y formación académica. A este respecto, las teorías de administración, psicología, pedagogía y sociología han aportado ideas significativas en cuanto a la naturaleza de los procesos de capacitación. Por tanto, los individuos, las empresas y los gobiernos deben mantenerse siempre en constante mejoramiento, evaluación y seguimiento de los procesos de formación y capacitación requeridos para mantener estándares de calidad y eficiencia apropiados.

En un mundo caracterizado por mercados abiertos y globalizados, la capacitación es una responsabilidad compartida por individuos, escuelas, universidades, empresas y gobiernos. De igual manera, la inversión en recursos, la ejecución, la capacitación y el seguimiento de los procesos de formación deben ser un esfuerzo combinado del sector público, el privado y la academia. Por supuesto, no se debe olvidar el rol del Estado que, a través de los canales pertinentes, debe establecer políticas públicas que apoyen y financien estos procedimientos educativos. De esta forma, los procesos se constituyen en una parte importante del desarrollo e incremento de la calidad de vida de los miembros de una sociedad.

En el ámbito costarricense, estas herramientas tecnológicas pueden ofrecer un gran aporte en la actualización que tanto se necesita en muchas instituciones del país. Las TIC pueden, por 
ejemplo, servir como medio para capacitar a todo el personal de una entidad tan grande como la Caja Costarricense del Seguro Social sin que el personal deba desplazarse y recibir la capacitación en un tiempo relativamente corto y a un costo mucho menor. Esto permitiría la implementación de expedientes digitales en el área de la salud, los cuales favorecerían la integración de la información y agilizarían el diagnóstico y tratamiento más oportuno de los pacientes que acuden ya sea a un centro de salud local o a diversos especialistas en un hospital.

Por tanto, el reto de los procesos de capacitación ayudados por TIC en nuestro país consiste en involucrar a la mayor cantidad de sectores en la formación del recurso humano con miras tanto al desarrollo de habilidades tecnológicas como a nuevos procesos productivos. Asimismo, en los procesos de capacitación, el financiamiento es un requisito indispensable para la inserción del recurso humano en las empresas, en el mercado nacional e internacional, en el mediano y largo plazos. Lo anterior demanda cambios en la cultura laboral para priorizar el potencial humano en grupos vulnerables de la población cuyas posibilidades de formación son más limitadas.

Este cambio es especialmente importante de enfatizar en Costa Rica cuando hablamos de los grupos más vulnerables de la población para quienes las posibilidades de formación se ven mucho más limitadas. En este sentido, los procesos de capacitación ayudados por TIC denotan claramente cómo estas herramientas pueden cumplir un rol fundamental al cerrar brechas en las oportunidades de actualización profesional que las instituciones públicas y privadas pueden ofrecer a los trabajadores costarricenses en todos los sectores de la sociedad.

Las tres experiencias de capacitación a las que se refiere este estudio así lo demuestran: (1) utilización de las TIC en la formación y desarrollo del capital humano: la experiencia de la Empresa de Telecomunicaciones de Cuba, S.A. (Etecsa); (2) capacitación docente en recursos de comunicación mediada por TIC y capacitación para integrar TIC al currículo de la cátedra universitaria: CHeTIC y PresTIC: dos cursos en línea desarrollados para la Facultad de Ciencias Económicas y Empresariales de la Universidad Morón de Buenos Aires; y (3) implementación del programa de capacitación docente en el correcto uso de las TIC en la Universidad Og Mandino en el año 2010 por medio de la aplicación de la Metodología Pacie. Las tres experiencias evidencian el éxito en la capacitación laboral con TIC.

Por último, pero no menos importante, así como las experiencias expuestas ayudaron a solventar los distintos problemas de capacitación de las empresas en que se implementaron, en nuestro país, con las TIC, sería posible ofrecer nuevas oportunidades para el desarrollo profesional y personal de los individuos, quienes serían más competitivos y exitosos en el mercado laboral. Finalmente, es importante señalar también que el debido seguimiento de las cuatro etapas que conlleva un programa de capacitación de esta índole será crucial en la consecución de este objetivo. 


\section{Bibliografía}

Aguilar-Morales, J. El Diagnóstico de Necesidades de Capacitación. Network de Psicología Internacional. México: Asociación Oaxaqueña de Psicología A.C., 2010.

Alemán, L. y Gómez, M. Administración de proyectos de capacitación basados en tecnología. México: ITESM, 2011. Alonso, A. y Zamora, M. Utilización de las Tecnologías de la Información y las Comunicaciones (TICs) en la Formación y Desarrollo del Capital Humano: La Experiencia de la Empresa de Telecomunicaciones de Cuba, S.A (ETECSA). Trabajo presentado en Virtual Brasil, 2007.

Bertalanffy, L. General System theory: Foundations, Development, Applications. Nueva York: George Braziller, edición revisada de 1976: ISBN 0-8076-0453-4, 1969.

Bobbit, J. The Curriculum: a summary of the development concerning the theory of the curriculum. Boston: Houghton Mifflin Company, 1918.

Castañón, R. Implementación del Programa de Capacitación Docente en el Correcto Uso de las TIC's en la Universidad Og Mandino de la Ciudad de Quito, Ecuador, a través de la Aplicación de la Metodología PACIE. Disponible en http://es.scribd. com/doc/34671449/FaseInvestigacionGrupoNFatlaScrib, 2010.

Coriat, B. El taller y el cronómetro. Ensayo sobre el taylorismo, el fordismo y la producción en masa. México: Siglo XXI, 1991.
Chiavenato, A. Introducción a la teoría general de la administración. México: Mc Graw-Hill, 1985.

Curso Chetic. Cursos del Departamento de Educación a distancia. Disponible en http://campus.unimoron.edu.ar/ course/view.php?id=1591\&section=1, 2015.

Oñate, L. La Metodología PACIE. Disponible en http://iuetaebvirtual.wikispaces.com/file/view/22234756-LaMetodologia-Pacie.pdf, 2009.

Taylor, F. W. The Principles of Scientific Management. Nueva York y Londres: Harper \& Brothers, 1911.

Westbrook, R. B. Thinkers on Education in Electronic Format: John Dewey. Perspectivas: revista trimestral de educación comparada. París: Unesco. Oficina Internacional de Educación, vol. XXIII, n. ${ }^{\circ}$ 1-2, 1993, págs. 289-305, 1993.

Zega, M. y Moreno, M. (2011). Experiencia de capacitación docente en recursos de comunicación mediada por TIC y capacitación para integrar TIC al currículo de la cátedra universitaria: CHeTIC y PresTIC. Dos cursos en línea desarrollados para la Facultad de Ciencias Económicas y Empresariales de una universidad privada de Buenos Aires. Trabajo presentado al concurso Premio ABA 2010/2011. Disponible en http:// www.aba-argentina.com/prensa/ gacetillas/se-entregaron-las-distinciones-el-premio-aba-201011-a-laeducacion/, 2011. 